\title{
Isolation of mip (Microtubule-Interacting Protein) Mutations of Aspergillus nidulans
}

\author{
CLIFFORD F. WEIL, C. ELIZABETH OAKLEY, AND BERL R. OAKLEY* \\ Department of Microbiology, The Ohio State University, Columbus, Ohio 43210
}

Received 19 December 1985/Accepted 22 April 1986

\begin{abstract}
We identified four mutations in two previously undescribed loci involved in microtubule function in Aspergillus nidulans as extragenic suppressors of benA33, a heat-sensitive $\beta$-tubulin mutation. Three of the four mutations map to a locus closely linked to riboB on linkage group VIII; we designated this locus mipA (for microtubule-interacting protein). We were not able to map the remaining suppressor because of chromosomal rearrangements. However, since it recombines with riboB at a significantly higher frequency than the mipA alleles, it is unlikely to be in mipA; thus, we designated it mipB1. The mip mutations are not allelic to the previously identified loci that encode $\alpha$ - and $\beta$-tubulin, and it is likely that mipA and mipB encode previously unidentified nontubulin proteins involved in microtubule function. Each of the mip mutations suppresses the heat sensitivity conferred by benA33 and suppresses the blockage of nuclear division and movement conferred by this mutation at high temperatures. Interactions between mipA and benA are allele specific. All of the mipA mutations are cryptic in a wild-type benA background but cause cold sensitivity in combination with benA33. These mutations also confer cold sensitivity in combination with benA31 and benA32 and reduce the resistance conferred by these mutations to the antimicrotubule agent benomyl but do not suppress the heat sensitivity conferred by these alleles. Finally, the mipA alleles suppress the heat sensitivity conferred by benA11, benA17, and benA21 but do not confer cold sensitivity in combination with these alleles.
\end{abstract}

The identification of the functional components of microtubules is a prerequisite for determining the mechanisms of microtubule-based motility. Although the major components of microtubules, $\alpha$ - and $\beta$-tubulin, have been known for more than a decade, the identities of other proteins required for microtubule function remain undetermined.

Microtubules have been the subject of intense biochemical study which has led to the identification of several proteins that copurify with $\alpha$-and $\beta$-tubulin through several rounds of microtubule assembly and disassembly. These microtubuleassociated proteins (MAPs) are the subject of an excellent recent review (13). An intrinsic limitation of biochemical studies of MAPs is that copurification of proteins with $\alpha$ - and $\beta$-tubulin does not demonstrate unambiguously that they are essential to microtubule function or even that they are associated with microtubules in vivo. Indeed, recent evidence suggests that at least one MAP is not associated with microtubules in vivo $(1,3,10)$.

We chose to identify nontubulin components of microtubules by a genetic approach-isolating mutations that affect microtubule function in vivo, characterizing these mutations, and identifying the products of the mutant genes. We attempted to isolate such mutations as revertants of a heat-sensitive $\left(\mathrm{hs}^{-}\right) \quad \beta$-tubulin mutation of Aspergillus nidulans. This approach is based on the demonstration in bacteriophage P22 that reversion of some hs ${ }^{-}$mutations is often due to mutations in other genes, the products of which interact physically with the original mutant protein (4). In addition, these extragenic suppressor mutations often cause cold sensitivity. Morris et al. (6) demonstrated that this approach can be used to isolate mutations in microtubule proteins of $\boldsymbol{A}$. nidulans. They isolated extragenic suppressors of $\mathrm{hs}^{-}$benA ( $\beta$-tubulin) mutations and were able to identify among them mutations in an $\alpha$-tubulin structural

\footnotetext{
* Corresponding author.
}

gene which they designated $t u b A$. Thomas et al. (12) have recently reported the isolation of $\mathrm{hs}^{-}$revertants of a coldsensitive $\left(\mathrm{cs}^{-}\right) \beta$-tubulin mutation of Saccharomyces cerevisiae. Among them were a single $\mathrm{hs}^{-} \beta$-tubulin mutation and 26 extragenic suppressors in 16 complementation groups. We took a similar approach, isolating large numbers of revertants of benA33, a $\beta$-tubulin mutation of $A$. nidulans that confers heat sensitivity and resistance to the antimicrotubule agent benomyl (7). This mutation inhibits mitosis and translocation of organelles at restrictive temperature but does not inhibit microtubule assembly (7). Substantial evidence suggests that this mutation hyperstabilizes microtubules such that they do not disassemble normally at high temperatures $(2,7)$. Among these revertants, we have identified benA and $t u b A$ mutations that confer conditional lethality (8; B. R. Oakley, C. E. Oakley, and J. E. Rinehart, submitted for publication). Here, we report the isolation and initial characterization of suppressor mutations in previously unidentified loci involved in microtubule function.

\section{MATERIALS AND METHODS}

Strains. Genotypes and sources of the A. nidulans strains used are listed in Table 1.

Mutagenesis. Mutagenesis of BRO2 conidiospores by UV irradiation and isolation of revertants have been previously described (8). Mutagenesis resulted in $25 \%$ survival.

Media. Complete and minimal media used were those described previously (8). Testing for the facB marker was on minimal medium in which $0.1 \mathrm{M}$ sodium acetate was substituted for glucose. Benomyl (technical grade, $98 \%$ pure) was a generous gift from Du Pont Co., Wilmington, Del.

Light microscopy. Fixation of germlings with glutaraldehyde, staining of nuclei and mitochondria with $4^{\prime}, 6-$ diamidino-2-phenylindole, and observation of nuclear and mitochondrial movement were done as previously described $(8,9)$. 


\section{RESULTS}

As reported elsewhere (8; Oakley et al., submitted), 2,604 non-heat-sensitive $\left(\mathrm{hs}^{+}\right)$revertants of strain $\mathrm{BRO2}$ were isolated and tested for cold sensitivity. A total of 128 revertants were $\mathrm{cs}^{-}$. To determine whether reversion in each case was due to a mutation at the benA locus or to an extragenic suppressor, each $\mathrm{cs}^{-}$revertant was crossed with a strain carrying a wild-type benA allele $\left(b_{e n A^{+}}\right)$. A total of 78 revertants were crossed successfully. In 44 crosses, reversion was closely linked to benA; in 34 cases, reversion was due to an extragenic suppressor.

To determine, in the revertants carrying extragenic suppressors, whether cold sensitivity was due to the suppressor or to a mutation that occurred coincidentally during mutagenesis, we examined progeny from crosses between $\mathrm{BRO} 2$ revertants and benA ${ }^{+}$strains for cosegregation of cold sensitivity and suppression of the heat sensitivity conferred by benA33. Segregants carrying benA33 can be identified by their resistance to the antimicrotubule agent benomyl (7). If cold sensitivity and suppression of heat sensitivity are caused by the same mutation, all progeny carrying ben $A 33$ and the suppressor of benA33 will be $\mathrm{cs}^{-} \mathrm{hs}^{+}$and benomyl resistant (ben'). If cold sensitivity and suppression of heat sensitivity are caused by different mutations, some hs ${ }^{+}$ben $^{\mathrm{r}}$ progeny will not be cold sensitive $\left(\mathrm{cs}^{+}\right)$. Crosses of $\mathbf{3 0}$ revertants carrying extragenic suppressors with ben $^{+}$ strains were examined. Crosses of 10 of these revertants yielded substantial numbers of $\mathrm{cs}^{+} \mathrm{hs}^{+}$ben $^{\mathrm{r}}$ progeny, but crosses of the remaining 20 revertants yielded no such segregants among 400 progeny tested from each cross, suggesting that cold sensitivity and suppression of heat sensitivity are very closely linked and almost certainly caused by the same mutation (Oakley et al., submitted).

Two revertants, BRO2RC21 and BRO2RP52, carried additional coincidental mutations. BRO2RC21 carried a mutation that reduced colony size at all temperatures, and BRO2RP52 carried a weak nutritional requirement. For subsequent studies, segregants that did not carry these additional mutations were used.

Morris et al. (6) have identified an $\alpha$-tubulin mutation, tubAl, that suppresses the $\mathrm{hs}^{-}$phenotype of benA33. We suspected that some of the extragenic suppressors might be mutations in tubA; consequently, we crossed each of the 20 revertants in which the benA33 suppressor causes cold sensitivity with strain LO196, which carries $t u b A 4$, another tubA allele that suppresses the hs ${ }^{-}$phenotype of benA33 (Oakley et al., submitted). If, in the revertants, suppression of the $\mathrm{hs}^{-}$phenotype is not due to a mutation of the tubA gene, crossovers between tubA4 and the suppressor carried by the revertant would produce benA33 tubA $A^{+}$suppressor ${ }^{+}$ progeny. Such progeny would be $\mathrm{cs}^{+} \mathrm{hs}^{-}$ben $^{\mathrm{r}}$. If the suppressor is a tubA mutation, such progeny would be extremely rare. Crosses of 16 revertants gave no more than $1 \mathrm{hs}^{-}$segregant among more than 400 tested. These revertants probably carry $t u b A$ mutations and have been reported elsewhere (Oakley et al., submitted). Crosses of the four remaining revertants (BRO2RA31, BRO2RC21, BRO2RP9, and BRO2RP52) gave significant numbers $(\simeq 10 \%)$ of $\mathrm{cs}^{+} \mathrm{hs}^{-}$ ben $^{\mathrm{r}}$ progeny, demonstrating that suppression of the $\mathrm{hs}^{-}$ phenotype of benA33 is not due to a tubA mutation but to a mutation at another, previously undescribed, locus.

We attempted to determine how many loci were represented among these four suppressors by performing complementation tests with stable diploids and by crossing each revertant with the other three. Our efforts were unsuccessful
TABLE 1. A. nidulans strains used

\begin{tabular}{|c|c|c|}
\hline Strain & Genotype & Source \\
\hline BRO2 & $y A 2$ benA33 & B. R. Oakley \\
\hline BRO2RA31 & Revertant of BRO2 & This study \\
\hline BRO2RC21 & Revertant of BRO2 & This study \\
\hline BRO2RP9 & Revertant of $\mathrm{BRO} 2$ & This study \\
\hline BRO2RP52 & Revertant of BRO2 & This study \\
\hline L0196 & $\begin{array}{c}\text { yA2 wA3 adE20 pabaAl } \\
\text { proAl pyroA4 tubA4 }\end{array}$ & C. E. Oakley \\
\hline FGSC 154 & $\begin{array}{l}\text { adE20 biAl wA3 cnxE16 sC12 } \\
\text { methGl nicA2 lacAl choAl } \\
\text { chaAl }\end{array}$ & $\begin{array}{l}\text { Fungal } \\
\text { Genetics } \\
\text { Stock Center }\end{array}$ \\
\hline FGSC 442 & $\begin{array}{l}\text { facB101 riboB2 chaAl sE15 } \\
\text { nirA14 }\end{array}$ & $\begin{array}{l}\text { Fungal } \\
\text { Genetics } \\
\text { Stock Center }\end{array}$ \\
\hline FGSC 256 & $\begin{array}{l}\text { pabaAl wA3 argC3 facBlol } \\
\quad \text { riboB2 }\end{array}$ & $\begin{array}{l}\text { Fungal } \\
\text { Genetics } \\
\text { Stock Center }\end{array}$ \\
\hline FGSC 449 & acrA1 lysB5 sD85 cnxB2 & $\begin{array}{l}\text { Fungal } \\
\text { Genetics } \\
\text { Stock Center }\end{array}$ \\
\hline FGSC 484 & $\begin{array}{l}\text { acrA1 lysB5 sD85 fwA2 } \\
\text { facC102 }\end{array}$ & $\begin{array}{l}\text { Fungal } \\
\text { Genetics } \\
\text { Stock Center }\end{array}$ \\
\hline LO427 & yA2 benA33 facB101 riboB2 & This study \\
\hline K5A & yA2 benA33 ornB 7 pabaAl & D. Kirsch \\
\hline LO413 & benA33 mipAl chaAl riboB2 & This study \\
\hline LO428 & benA33 chaAl riboB2 facB101 & This study \\
\hline BEN1 & biAl benA3 & J. M. Van Tuyl \\
\hline BEN2 & biAl benA4 & J. M. Van Tuyl \\
\hline BEN9 & biAl benAll & J. M. Van Tuyl \\
\hline BEN14 & biAl benAl6 & J. M. Van Tuyl \\
\hline BEN15 & biAl benAl7 & J. M. Van Tuyl \\
\hline BEN17 & biAl benAl9 & J. M. Van Tuyl \\
\hline BEN19 & biAl benA21 & J. M. Van Tuyl \\
\hline BEN30 & wA3 pyroA4 benA3I & B. R. Oakley \\
\hline BEN31 & wA3 pyroA4 benA32 & B. R. Oakley \\
\hline
\end{tabular}

despite repeated attempts. No two revertants formed heterokaryons, and no crosses produced hybrid cleistothecia.

To surmount these problems, we attempted to map each suppressor to linkage group and, subsequently, to locus. To map the suppressors to linkage group, we constructed diploids from strain FGSC 154, which carries a marker on each linkage group, and segregants carrying benA33 and each suppressor. Segregants of revertant $\times b_{e n A^{+}}$crosses were used instead of revertants because the revertants did not carry the necessary nutritional markers. We induced random chromosome loss by treatment with chloral hydrate (11). Two of the suppressors mapped to linkage group VIII, and a third suppressor appeared to map to linkage group III or VIII. The data for the fourth suppressor (isolated in BRO2RP9) suggest that it is on linkage group VI or VIII, but an apparent translocation between these linkage groups, as well as additional chromosomal rearrangements, has prevented definitive assignment to linkage group. Because these rearrangements greatly complicate genetic analysis, we concentrated our efforts on the other three suppressors.

To map these suppressors to locus, we crossed strains carrying each suppressor with strains FGSC 256, FGSC 442, FGSC 449, and FGSC 484, all of which are multiply marked on linkage group VIII (Table 1). All three mutations were found to be closely linked to the $f a c B$ and $r i b o B$ genes, more loosely linked to the $\arg C$ gene, and unlinked to the $f w A$, facC, $\operatorname{cn} x B$, chaA, $s E$, and nirA genes (data not shown). The data suggest that all three mutations may lie within the same 


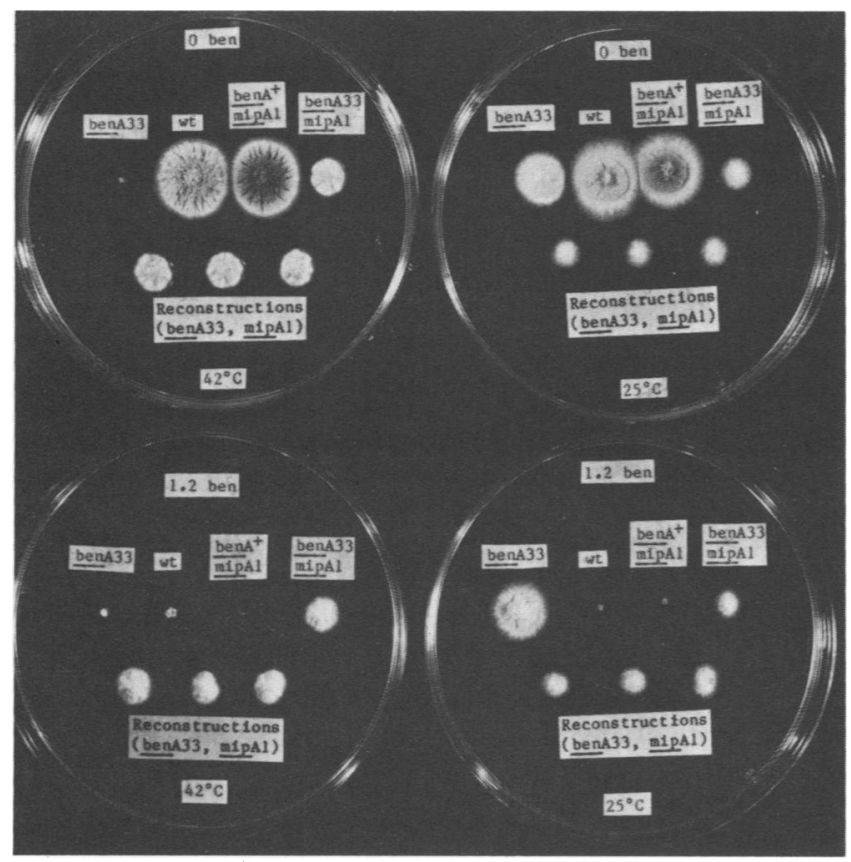

FIG. 1. Effects of benomyl concentration and temperature on strains carrying benA33 or mipAl or both. The temperatures of incubation are shown at the bottom of each plate; the concentrations of benomyl used are shown at the top of each plate. The strain at the left end of the top row on each plate carries benA33 and mipA ${ }^{+}$and is $\mathrm{hs}^{-}$and benomyl resistant. The wild-type strain is $\mathrm{hs}^{+} \mathrm{Cs}^{+}$and benomyl sensitive. The strain carrying $b e n A^{+}$and $m i p A l$ is, like the wild-type strain, $\mathrm{hs}^{+} \mathrm{cs}^{+}$and benomyl sensitive. The revertant carrying benA33 and mipAl is less heat sensitive than the strain carrying benA33 and mip $A^{+}$and is somewhat cold sensitive. The bottom row of each plate consists of three progeny of a cross between a strain carrying benA33 and $\mathrm{mipA}^{+}$and a strain carrying ben $^{+}$and mipAl. These progeny have the same phenotype as the revertant.

gene. We designated this putative gene mipA (microtubuleinteracting protein). We designated the suppressor alleles mipA1, mipA2, and mipA3 (isolated from BRO2RA31, BRO2RC21, and BRO2RP52, respectively).

Three-point crosses involving the $f a c B$ and riboB mutations and each mipA mutation yielded few or no recombinants between the mip $A$ alleles and the riboB gene among over $\mathbf{4 0 0}$ progeny tested in each case. To determine the order of these three genes and the distance between the mipA alleles and the riboB2 mutation, we plated large numbers of ascospores on medium containing benomyl and lacking riboflavin and incubated these ascospores at $25^{\circ} \mathrm{C}$. These conditions selected for $\mathrm{mipA}^{+}$riboB ${ }^{+}$progeny obtained if crossing over occurred between mipA and riboB. We then tested the colonies which arose to determine their facB phenotype.

Of $1.26 \times 10^{4}$ segregants from the cross BRO2RA31 $\times$ FGSC 442,41 were $\mathrm{cs}^{+}$ribo $^{+}$. Of these 41 segregants, 39 were fac ${ }^{-}$, indicating that mipAl maps 0.325 centimorgans (cM) to the left of riboB2. Of $1.49 \times 10^{4}$ progeny from the

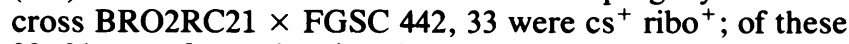
33,31 were fac $^{-}$, showing that $\operatorname{mip} A 2$ maps $0.221 \mathrm{cM}$ to the left of riboB2. Of $9.5 \times 10^{3}$ spores from the cross BRO2RP52

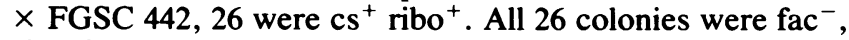
showing that mipA3 maps $0.274 \mathrm{cM}$ to the left of riboB2.

Although chromosomal rearrangements did not allow us to map the suppressor in BRO2RP9 precisely, recombination between this suppressor and riboB2 occurred with a significantly higher frequency than for any of the mipA alleles $(\geq 6.9 \%)$. This value represents a minimum estimate of the map distance between riboB and the suppressor in BRO2RP9 since the chromosomal rearrangements can be expected to inhibit recombination. Therefore, the suppressor isolated in BRO2RP9 is unlikely to be allelic to the mipA mutations; thus, we designated it mipB1.

Phenotypes of mip mutations. To determine whether any of the four suppressor alleles conferred cold sensitivity in a ben $A^{+}$background, we tested progeny from crosses between the $\mathrm{BRO} 2$ revertants and the $b e n A^{+}$strains for the presence of $\mathrm{cs}^{-} \mathrm{hs}^{+}$benomyl-sensitive $\left(\right.$ben $\left.^{\mathrm{s}}\right)$ progeny. Since benomyl resistance is conferred by benA33 but not by ben $^{+}$, all ben ${ }^{\text {s }}$ progeny will carry ben $A^{+}$. We recovered several hundred progeny from plates containing no benomyl which were incubated at $42^{\circ} \mathrm{C}$. There is a significant growth advantage for benA $A^{+}$spores under these conditions.

For each of the mipA alleles, all ben ${ }^{\text {s }}$ colonies were $\mathrm{cs}^{+}$ $\mathrm{hs}^{+}$(data not shown). The absence of benA ${ }^{+} \mathrm{cs}^{-}$progeny suggests that each of the mipA alleles is either cryptic or lethal in a ben $A^{+}$background. The close linkage of mipA to riboB allowed us to determine which of these possibilities is correct.

We first crossed each revertant carrying a mipA mutation to FGSC 442, which carries facB101 and riboB2. Since each mutant mipA allele is closely linked to riboB ${ }^{+}$, the presence of substantial numbers of ben ${ }^{\mathrm{s}}$ ribo ${ }^{+}$spores indicates that the mipA mutation is cryptic in a ben $A^{+}$background. The absence of such progeny would suggest that the $\operatorname{mip} A$ allele is lethal in a benA ${ }^{+}$background. Each cross produced significant numbers of ben ${ }^{\mathrm{s}}$ ribo ${ }^{+}$progeny. To confirm the presence of the mipA mutant alleles in these bens ${ }^{s}$ ribo $^{+}$ progeny, we crossed three such progeny from each cross to strain LO427 (yA2 benA33 facB101 riboB2). Each cross produced progeny which were $\mathrm{cs}^{-} \mathrm{hs}^{+} \mathrm{ben}^{\mathrm{r}}$, greater than $99 \%$ of which were $r i b o B^{+}$. These progeny have the same phenotype as the original hs ${ }^{+}$revertants (Fig. 1). Thus, each of the mipA mutations is cryptic in a ben $A^{+}$background but $\mathrm{Cs}^{-}$in a benA33 background.

In a BRO2RP9 $\times$ ben $A^{+}$strain cross, ben $A^{+} \mathrm{cs}^{-}$progeny were recovered at a frequency of approximately $20 \%$. This result suggests that mipBI confers cold sensitivity even in the absence of the benA33 mutation, but this $\mathrm{cs}^{-}$phenotype cannot yet be rigorously assigned to the mipBl mutation because of the presence of chromosomal rearrangements.

Analysis of mip $^{-}$benA33 double mutants with respect to

TABLE 2. Effects of mip mutations on nuclear division, nuclear migration, and mitochondrial movement ${ }^{a}$

\begin{tabular}{|c|c|c|c|c|c|c|}
\hline \multirow[t]{2}{*}{ Genotype } & \multicolumn{2}{|c|}{$\begin{array}{l}\text { \% Nuclear } \\
\text { division at } \\
\left({ }^{\circ} \mathrm{C}\right):\end{array}$} & \multicolumn{2}{|c|}{$\begin{array}{l}\text { \% Nuclear } \\
\text { movement at } \\
\left({ }^{\circ} \mathrm{C}\right):\end{array}$} & \multicolumn{2}{|c|}{$\begin{array}{c}\% \\
\text { Mitochondrial } \\
\text { movement at } \\
\left({ }^{\circ} \mathrm{C}\right):\end{array}$} \\
\hline & 25 & 37 & 25 & 37 & 25 & 37 \\
\hline $\operatorname{benA}^{+} \operatorname{mipA}^{+}$ & 97 & 100 & 98 & 99 & 86 & 97 \\
\hline benA33 mipA ${ }^{+}$ & 94 & 58 & 88 & 53 & 99 & 100 \\
\hline benA33 mipAl & 82 & 96 & 84 & 84 & 76 & 96 \\
\hline benA33 mipA2 & 99 & 98 & 93 & 82 & 99 & 100 \\
\hline benA33 mipA3 & 94 & 99 & 89 & 89 & 98 & 95 \\
\hline benA33 mipB1 & 88 & 99 & 94 & 84 & 100 & 100 \\
\hline
\end{tabular}

${ }^{a}$ The values shown are the percentages of germlings in which these processes occurred. Sample size for each value was 100 . Details of fixation, staining, and scoring have been reported previously $(8,9)$. 
other phenotypes showed that all three mipA alleles suppressed the inhibition of nuclear division and nuclear movement conferred by benA33 at high temperatures (Table 2). At $25^{\circ} \mathrm{C}$, the restrictive temperature of the mip $A^{-}$benA33 double mutants, nuclear division and nuclear movement were not significantly reduced, perhaps reflecting the leakiness of the $\mathrm{cs}^{-}$phenotype in each of the double mutants.

To determine whether the mipA mutations are allelespecific suppressors, we constructed a series of mipA benA double mutants by using different ben $A$ alleles. In all cases, mipA1, mipA2, and mipA3 gave identical results. The mipA mutations suppressed the hs ${ }^{-}$phenotype of the benAll, benA17, and benA21 mutations (6; J. M. Van Tuyl, Ph.D. thesis, Agricultural University, Wageningen, The Netherlands, 1977) but did not confer cold sensitivity in the presence of these alleles (Fig. 2). With the benA31 and benA32 mutations (B. R. Oakley and N. R. Morris, unpublished data), the mipA mutations did not significantly suppress heat sensitivity but did confer cold sensitivity and also lowered the level of benomyl resistance conferred by benA31 and benA32 (Fig. 2). mipA mutations also appeared to reduce the benomyl resistance conferred by benA33 (results not shown). ben ${ }^{\mathrm{r}} \mathrm{hs}^{+}$alleles benA3 and benA4 and benomyl-supersensitive alleles benAl6 and benA19 (Van Tuyl, Ph.D. thesis) were not significantly affected by any of the mipA alleles (data not shown).

Dominance relationships of mipA mutations. Stable diploids of $A$. nidulans can normally be obtained by maintaining heterokaryons, formed from haploids carrying complementing auxotrophic markers, on selective medium for several weeks. To determine whether the mipA mutations are dom-

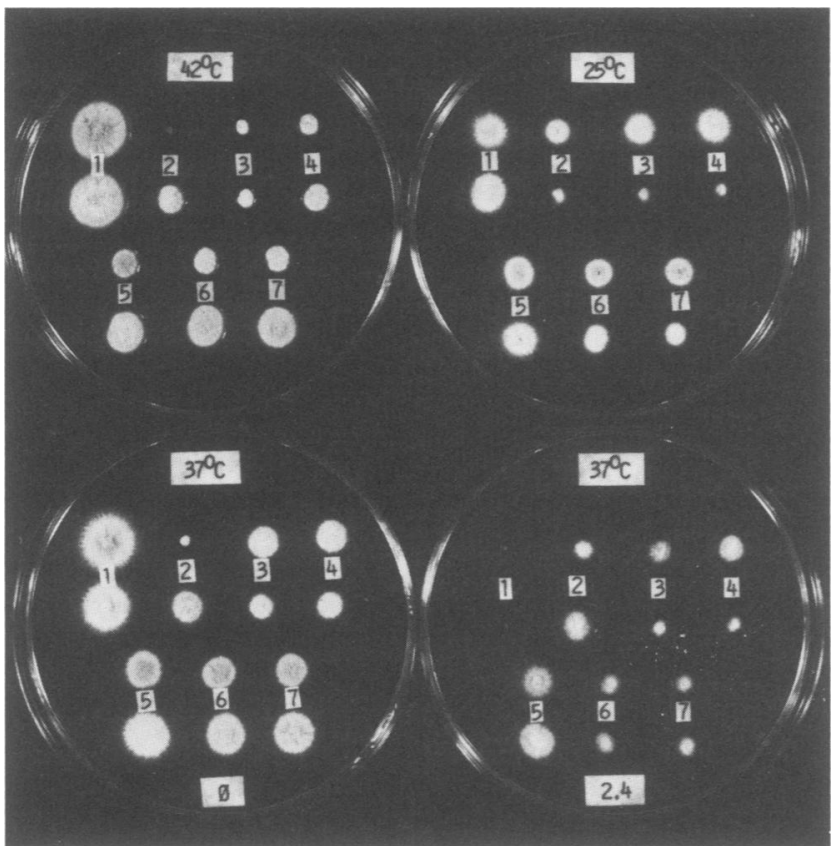

FIG. 2. Interactions between benA alleles and mipA alleles. Temperatures of incubation are given at the top of each plate. Benomyl concentrations are given at the bottom of the two bottom plates. The top two plates contain no benomyl. The colonies are arranged in rows, with the colony above each number carrying $\mathrm{mipA}^{+}$and the colony below each number carrying mipAl. The colonies above and below each number carry the same benA allele: 1 , benA ${ }^{+} ; 2$, benA $33 ; 3$, benA $31 ; 4$, benA $32 ; 5$, benA11; 6, benA17; 7 , benA21.

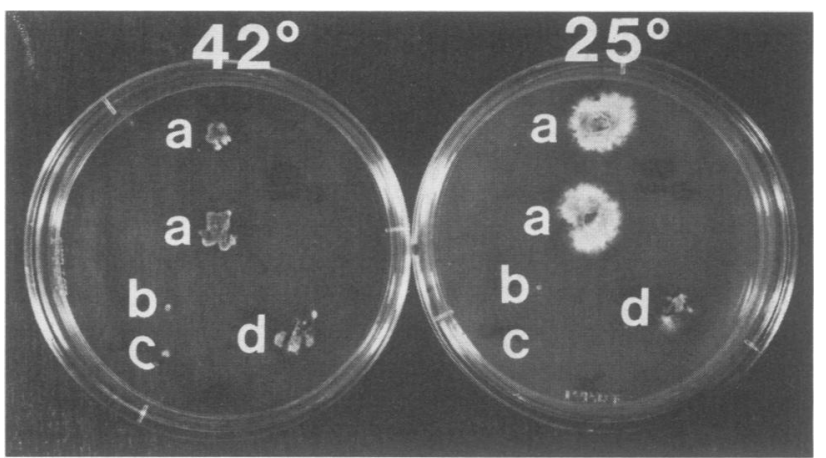

FIG. 3. Dominance relationship of mipAl. The plate at the left was incubated at $42^{\circ} \mathrm{C}$; the plate at the right was incubated at $25^{\circ} \mathrm{C}$. Colonies are designated by letters to their left. The two colonies designated by the letter a are identical heterokaryons formed from two strains, one carrying benA33 and $\mathrm{mipA}^{+}$and the other carrying benA33 and $\mathrm{mipAl}$. These heterokaryons grew at $25^{\circ} \mathrm{C}$ but not at $42^{\circ} \mathrm{C}$, indicating that the mutation is recessive. The letters $b$ and $c$ designate the two parental strains which did not grow in this case because they carry nutritional markers which do not allow growth on the minimal medium used. The letter $d$ designates a control heterokaryon constructed from two strains carrying benA33 and mip $A^{+}$.

inant or recessive, we attempted to make diploids between benA33 mipA $^{-}$strains and benA33 mipA ${ }^{+}$strains. Heterokaryons formed at $28^{\circ} \mathrm{C}$ but not at 25 or $42^{\circ} \mathrm{C}$, the restrictive temperatures of each of the respective parents. In all three cases, the heterokaryons were $\mathrm{hs}^{-} \mathrm{cs}^{+}$, compared with wild-type and benA33 $\mathrm{mipA}^{+} /$benA33 $\mathrm{mipA}^{+}$controls (Fig. 3). These data suggest that all three mipA mutations are recessive. In all heterokaryons in which both parents carried the benA33 mutation, stable diploids did not form, even after 8 weeks, although the heterokaryons persisted if subcultured weekly. Streaking the heterokaryons to single colonies on nonselective medium after this time produced haploids of the expected parental types.

\section{DISCUSSION}

We isolated, among extragenic suppressors of benA33, four mutations at two new loci. Three of the suppressor mutations map to less than $0.4 \mathrm{cM}$ to the left of the riboB2 mutation on linkage group VIII. The close linkage of these mutations suggests strongly that they are allelic, and we have assigned them to a new gene, mipA. Reconstruction experiments demonstrated that these mutations are cryptic in a benA $A^{+}$background but confer a $\mathrm{cs}^{-} \mathrm{hs}^{+}$phenotype in the presence of the normally $\mathrm{cs}^{+} \mathrm{hs}^{-}$mutation, benA33.

Despite the remarkable phenotypic similarities of the three mipA alleles, we are confident that they are the result of three separate mutational events. The mutagenesis was of uninucleate conidiospores, each revertant was isolated from a separate plate, and two of the three carried different coincidental mutations.

Although each of the mipA mutations is closely linked to the riboB2 mutation, we do not believe that they are mutations in the riboB gene itself for two reasons. None of the mipA alleles confers riboflavin auxotrophy, and the presence of $\mathrm{Cs}^{+} \mathrm{hs}^{-}$ribo $^{-}$progeny from crosses of the original ribo $^{+}$ revertants to riboB $B^{-}$strains demonstrates that riboflavin auxotrophy does not suppress the hs ${ }^{-}$phenotype of benA33. However, it is still possible that the mipA mutations are in 
the riboB gene but that they do not affect riboflavin biosynthesis.

The fact that the mip mutations suppress the heatsensitive blockage of microtubule function conferred by benA33 demonstrates that the products of these genes interact with the $\beta$-tubulin encoded by the benA gene and are involved in the functioning of microtubules. However, it should be pointed out that whereas the most obvious type of interaction between the mip gene products and $\beta$-tubulin is a direct, physical one, such as the interaction between a MAP and tubulin, two other equally interesting modes of interaction are possible and consistent with our data. One is that mip genes encode products that interact specifically but indirectly with $\beta$-tubulin. It is likely that there are classes of proteins that are essential to microtubule function but that do not interact physically with microtubules. To choose one of many possible examples, such proteins might be involved in a posttranslational modification that affects the functioning of a MAP. Another possibility is that one or both of the mip genes encode a protein involved in regulating the cellular environment in a way that affects microtubule function. A mip product could be involved in the regulation of $\mathrm{Ca}^{2+}$ levels or $\mathrm{pH}$, for example.

Our results do not allow us to distinguish unambiguously among these possibilities, but they suggest that, for mipA, regulating the cellular environment is the least likely possibility. If mipA mutations caused significant alterations in the cellular environment, one would expect that they would have some effects on phenomena that are not microtubule mediated. Since the mipA mutations have no detectable effects in a ben $A^{+}$background, the alterations that they cause must be very subtle or very specific. The fact that different combinations of cold sensitivity, heat sensitivity, and benomyl resistance can be constructed by combining different benA alleles with the same mipA allele indicates that the interactions between the products of mipA and benA are allele specific. This specificity can be explained if the interactions between the mipA gene product and the $\beta$ tubulin encoded by benA are specific. For example, a mutation in a MAP that affected a site where the MAP binds to $\beta$-tubulin might cause the mutant MAP to bind with different affinities to $\beta$-tubulins encoded by different benA alleles, thus giving different phenotypes. It is much more difficult to explain how general alterations in the cellular environment could produce such allele-specific interactions.

The specificity of the interactions between the mipA and ben $A$ mutations also provides an explanation for the differences between our results and those of Thomas et al. (12). Thomas et al. (12) found, among hs ${ }^{-}$revertants of a $\mathrm{cs}^{-}$ $\beta$-tubulin mutation of $S$. cerevisiae, a single intragenic suppressor and 26 extragenic suppressors in 16 complementation groups. We found, among a significantly larger number of $\mathrm{cs}^{-}$revertants of an $\mathrm{hs}^{-} \beta$-tubulin mutation, that most carry intragenic suppressors and, although we also found a large number of extragenic suppressors, they define only three loci, $\operatorname{mip} A, \operatorname{mip} B$, and $t u b A$. It appears that the number of genes in which mutations can be isolated which both suppress the heat sensitivity of benA33 and confer cold sensitivity is small. These data also suggest that new mutations in previously undiscovered genes involved in microtubule function can be isolated more easily among revertants of other benA alleles than among additional revertants of benA33.

Two-dimensional gel electrophoresis has shown that, in $A$. nidulans, there is a $\beta$-tubulin produced in minor amounts in addition to the major $\beta$-tubulin encoded by benA (14). May et al. (5) have recently cloned the gene encoding this minor $\beta$-tubulin and designated it $t u b C$. Their evidence also suggests that there are no $\beta$-tubulin genes other than ben $A$ and $t u b C$. Since the benA33 allele is recessive with respect to heat sensitivity, a mutation that resulted in overproduction of the $t u b C \beta$-tubulin might overcome the heat sensitivity conferred by benA33. However, tubC has recently been mapped to linkage group I (G. May and N. R. Morris, personal communication), and since neither $\operatorname{mip} A$ nor mipB is on linkage group $\mathrm{I}$, neither can be $t u b C$. We believe it is more likely that $\operatorname{mip} A$ and $\operatorname{mip} B$ encode nontubulin proteins involved in microtubule function.

The results we report here do not permit the identification of the products of the mip genes or the determination of the function of these products. However, they do suggest an approach to the identification and characterization of the product of mipA which we are currently using. The close linkage of mipA to riboB suggests that, if $r i b o B$ can be cloned, it should be possible to identify a clone carrying mipA with a very short chromosome walk. The cloned mipA gene could be used to identify the mip $A$ gene product, and in vitro mutagenesis could be used to create the mipA mutations that have obvious phenotypes in ben $A^{+}$backgrounds. We have recently cloned a sequence complementing riboB2 (unpublished results), and we hope to be able to identify a clone carrying mipA soon.

\section{ACKNOWLEDGMENTS}

We are grateful to Janet Rinehart for excellent technical assistance.

This work was supported by Public Health Service grant GM31837 from the National Institutes of Health.

\section{LITERATURE CITED}

1. Asai, D. J., W. C. Thompson, L. Wilson, C. F. Dresden, H. Schulman, and D. L. Purich. 1985. Microtubule-associated proteins (MAPs): a monoclonal antibody to MAP1 decorates microtubules in vitro but stains stress fibers and not microtubules in vivo. Proc. Natl. Acad. Sci. USA 82:1434-1438.

2. Gambino, J., L. G. Bergen, and N. R. Morris. 1984. Effects of mitotic and tubulin mutations on microtubule architecture in actively growing protoplasts of Aspergillus nidulans. J. Cell Biol. 99:830-838.

3. Hill, A. M., R. Maunoury, and D. Pantaloni. 1981. Cellular distribution of the microtubule-associated proteins HMW $(350 \mathrm{~K}, 300 \mathrm{~K})$ by indirect immunofluorescence. Biol. Cell. 41:43-50.

4. Jarvik, J., and D. Botstein. 1975. Conditional-lethal mutations that suppress genetic defects in morphogenesis by altering structural proteins. Proc. Natl. Acad. Sci. USA 72:2738-2742.

5. May, G., J. Gambino, J. Weatherbee, and N. R. Morris. 1985. Identification and functional analysis of beta-tubulin genes by site-specific integrative transformation in Aspergillus nidulans. J. Cell Biol. 101:712-719.

6. Morris, N. R., M. H. Lai, and C. E. Oakley. 1979. Identification of a gene for alpha-tubulin in Aspergillus nidulans. Cell 16:437-442.

7. Oakley, B. R., and N. R. Morris. 1981. A beta-tubulin mutation that blocks microtubule function without blocking assembly. Cell 24:837-845.

8. Oakley, B. R., C. E. Oakley, K. S. Kniepkamp, and J. S. Rinehart. 1985. Isolation and characterization of cold-sensitive mutations at the benA beta-tubulin locus of Aspergillus nidulans. Mol. Gen. Genet. 201:56-64.

9. Oakley, B. R., and J. E. Rinehart. 1985. Mitochondria and nuclei move by different mechanisms in Aspergillus nidulans. J. Cell Biol. 101:2392-2398.

10. Sato, C., K. Nishizawa, H. Nakamura, Y. Komagoe, K. Shimada, R. Ueda, and S. Suzuki. 1983. Monoclonal antibody against 
microtubule-associated protein-1 produces immunofluorescent spots in the nucleus and centrosome of cultured mammalian cells. Cell Struct. Funct. 8:245-254.

11. Singh, M., and U. Sinha. 1979. Mitotic haploidization and growth of Aspergillus nidulans on media containing chloral hydrate. J. Cytol. Genet. 14:1-4.

12. Thomas, J. H., P. Novick, and D. Botstein. 1984. Genetics of the yeast cytoskeleton, p. 153-174. In G. G. Borisy, D. W. Cleve- land, and D. B. Murphy (ed.), Molecular biology of the cytoskeleton. Cold Spring Harbor Laboratory, Cold Spring Harbor, N.Y.

13. Vallee, R. B., G. S. Bloom, and W. E. Theurkauf. 1984. Microtubule-associated proteins: subunits of the cytomatrix. J. Cell Biol. 99:38-48.

14. Weatherbee, J. A., and N. R. Morris. 1984. Aspergillus contains multiple tubulin genes. J. Biol. Chem. 259:15452-15459. 